\title{
Alteration of epoxyeicosatrienoic acids in the liver and kidney of cytochrome P450 4F2 transgenic mice
}

\author{
BIJUN ZHANG ${ }^{1,2}$, GUANGRUI LAI ${ }^{1}$, XIAOLIANG LIU ${ }^{1}$ and YANYAN ZHAO ${ }^{1,2}$ \\ ${ }^{1}$ Department of Clinical Genetics, Shengjing Hospital of China Medical University, Shenyang, Liaoning 110004; \\ ${ }^{2}$ Department of Medical Genetics, China Medical University, Shenyang, Liaoning 110122, P.R. China
}

Received September 15, 2015; Accepted September 26, 2016

DOI: $10.3892 / \mathrm{mmr} .2016 .5962$

\begin{abstract}
Arachidonic acid (AA) can be metabolized into 20-hydroxyeicosatetraenoic acid (20-HETE) by $\omega$-hydroxylases, and epoxyeicosatrienoic acids (EETs) by epoxygenases. The effects of EETs in cardiovascular physiology are vasodilatory, anti-inflammatory and anti-apoptotic, which are opposite to the function to 20-HETE. However, EETs are not stable in vivo, and are rapidly degraded to the biologically less active metabolites, dihydroxyeicosatrienoic acids, via soluble epoxide hydrolase (sEH). Western blotting, reverse transcription-quantitative polymerase chain reaction and liquid chromatography tandem mass spectrometry were performed in order to determine target RNA and protein expression levels. In the present study, it was demonstrated that the disturbed renal 20-HETE/EET ratio in the hypertensive cytochrome P450 4F2 transgenic mice was caused by the activation of $\mathrm{sEH}$ and the repression of epoxygenase activity. In addition, 20-HETE showed an opposite regulatory effect on the endogenous epoxygenases in the liver and kidney. Given that 20-HETE and EETs have opposite effects in multiple disease, the regulation of their formation and degradation may yield therapeutic benefits.
\end{abstract}

\section{Introduction}

Arachidonic acid (AA) can be metabolized into hydroxyeicosatetraenoic acids (HETEs) by $\omega$-hydroxylases, and epoxyeicosatrienoic acids (EETs) by epoxygenases, respectively (1). Of the HETEs, 20-HETE is the most important isoform and is predominantly generated by the cytochrome P450 (CYP) 4A and 4F families, which have $\omega$-hydroxylase activities in the kidney and liver. EETs, including 8, 9-EET,

Correspondence to: Professor Yanyan Zhao, Department of Clinical Genetics, Shengjing Hospital of China Medical University, 36 San Hao Street, Shenyang, Liaoning 110004, P.R. China E-mail: yyzhao@sj-hospital.org; yyzhao@mail.cmu.edu.cn

Key words: 20-hydroxyeicosatetraenoic acids, epoxyeicosatrienoic acids, soluble epoxide hydrolase, $\omega$-hydroxylases, epoxygenases, cytochrome $\mathrm{P} 450$ 4F2 transgenic mice
11, 12-EET and 14, 15-EET, are formed by the CYP2C and $2 \mathrm{~J}$ families which have epoxygenase activity in the kidney and liver (2). Furthermore, EETs are rapidly degraded to the biologically less active metabolites, dihydroxyeicosatrienoic acids (DiHETEs) via soluble epoxide hydrolase (sEH) (3). 20-HETE has paradoxical functions in the regulation of blood pressure, with a prohypertensive effect by vasoconstriction and an anti-hypertensive effect by natriuresis (4). However, EETs have natriuretic, vasodilative, anti-inflammatory and anti-apoptotic properties (5-7). Therefore, the homeostasis of 20-HETE and EETs is important in the pathogenesis of hypertension.

In our previous study (8), it was reported that $C Y P 4 F 2$ transgenic mice showed hypertension and hyperglycemia, with increased intrarenal 20-HETE and decreased intrarenal EETs in the urine and kidney homogenate. Coincidentally, hypertensive Ren-2 transgenic rats exhibit increased synthesis of 20-HETE and deficient synthesis of EETs in the kidney, which may contribute to the development of hypertension (9). Similar disturbances in 20-HETE and EETs also occur in diabetic nephropathy induced by high glucose (10). The decrease of EETs may be caused by decreased generation via the CYP2C and CYP2J families or by increased degradation via $\mathrm{SEH}$ (11). Until now, whether 20-HETE affects the level of EETs remains to be fully elucidated. The aim of the present study was to investigate the metabolic mechanism underlying endogenous epoxygenases regulation by high levels of 20-HETE, and identify the cause of the reduced EETs in $C Y P 4 F 2$ transgenic mice.

\section{Materials and methods}

Sample collection. Blood samples were obtained from male patients with metabolic syndrome (MS) as the test group (45-56 years old; mean, 51 years old) and from a normal control group (45-54 years old; mean, 50 years old). Each group contained 20 samples, which were age-matched. The sample collection methods were approved by the Ethics Committee of Shengjing Hospital of China Medical University (Shenyang, China), and written informed consent was obtained from all patients prior to commencement of the investigation.

RNA purification and reverse transcription-polymerase chain reaction ( $R T-P C R)$ arrays. RNA was extracted from $1.5 \mathrm{ml}$ 
whole blood samples using the QIAamp ${ }^{\circledR}$ RNA Blood Mini kit (Qiagen GmbH, Hilden, Germany). Reverse transcription was performed with an $\mathrm{RT}^{2}$ First Strand kit (Qiagen $\mathrm{GmbH}$ ), which comprised an effective genomic DNA elimination step. The genomic DNA elimination reaction conditions were $42^{\circ} \mathrm{C}$ for $5 \mathrm{~min}$ and then on ice for $1 \mathrm{~min}$, then the reverse transcription reaction conditions were $42^{\circ} \mathrm{C}$ for 5 min followed by $95^{\circ} \mathrm{C}$ for $5 \mathrm{~min}$. The cDNA template was then detected using the Human Oxidative Stress RT2 Profiler $^{\mathrm{TM}}$ (Qiagen $\mathrm{GmbH}$ ) PCR Array in an ABI 7900HT detection system (Applied Biosystems; Thermo Fisher Scientific., Waltham, MA, USA). PCR reactions were performed to evaluate the expression of 84 genes associated with oxidative stress. The reaction conditions were $95^{\circ} \mathrm{C}$ for $10 \mathrm{~min}$ followed by 40 cycles of $95^{\circ} \mathrm{C}$ for $15 \mathrm{sec}$ and $60^{\circ} \mathrm{C}$ for $1 \mathrm{~min}$. Data analysis was performed using PCR Array Data Analysis software (Qiagen $\mathrm{GmbH}$ ).

Animals. CYP4F2 transgenic mice from the FVB/N strain were generated and characterized as previously described (12). The mice were fed with standard mouse chow and water ad libitum, and bred in a $12: 12 \mathrm{~h}$ light-dark cycle system at $23^{\circ} \mathrm{C}$. Experiments were performed on 14-20-week-old CYP4F2 transgenic mice weighing 24-33 g. Transgenic mice were matched by sex, weight and age with wild-type control mice, and each group contained 6 mice. All experiments conformed to the Guide for the Care and Use of Laboratory Animals (13).

Western blot analysis. The mice were sacrificed by cervical dislocation when they were 14-20-weeks old. Total protein from the livers and kidneys of the mice was extracted and the concentration was determined using the Bradford method. The denatured protein $(60 \mu \mathrm{g})$ was separated on a $10 \%$ SDS-PAGE gel by electrophoresis and transferred onto polyvinylidene difluoride membranes at $4^{\circ} \mathrm{C}$. The membranes were subsequently blocked in $5 \%$ skim milk in PBS, and incubated at $4^{\circ} \mathrm{C}$ overnight with the following primary antibodies: Rabbit anti-sEH (sc-25797; 1:200; Santa Cruz Biotechnology, Inc., Santa Cruz, CA, USA) and mouse polyclonal anti-GAPDH (10494-1-AP; 1:10,000; ProteinTech Group, Inc., Chicago, IL, USA). Subsequently, the membranes were incubated at room temperature for $1 \mathrm{~h}$ with polyclonal horseradish peroxidase-conjugated goat anti-rabbit IgG (ZB-2301; 1:4,000; ZSGB-BIO, Beijing, China) as the secondary antibody. The final detection reaction was performed with an ECL detection kit (Beyotime Institute of Biotechnology, Jiangsu, China). The blotted membranes were scanned, and the density of bands was quantified using Image J Lab Software, version 2.0.1 (Bio-Rad Laboratories, Inc., Hercules, CA, USA).

sEH activity assay. The liver and kidney tissues were homogenized in deionized water with $1 \mathrm{mM}$ PMSF, and cytosolic supernatants were obtained by centrifugation at $3,500 \mathrm{x} g$ for $10 \mathrm{~min}$ at $4^{\circ} \mathrm{C}$. The activity of sEH in the hepatic and renal homogenates was determined using Epoxy fluor 7 (Cayman Chemical Company, Ann Arbor, MI, USA) and the sEH inhibitor, trans-4-[4-(3-adamantan-1-ylureido)-cyclohex yloxy]-benzoic acid ( $t$-AUCB), as previously described (14). The sEH inhibitor, $t$-AUCB, was obtained from Professor Bruce D. Hammock (Department of Entomology and UCD
Cancer Research Center, University of California, Davis, CA, USA).

$R T$-quantitative PCR (RT-qPCR). Total RNA was isolated from the liver and kidney tissue using TRIzol reagent (Invitrogen; Thermo Fisher Scientific, Inc.), and reverse transcribed into cDNA $(1 \mu \mathrm{g})$ using a reverse transcription reagent kit (Promega Corporation, Madison, WI, USA). The PCR reactions were performed on the ABI 7,900 system (Applied Biosystems; Thermo Fisher Scientific, Inc.) in a $20 \mu 1$ SYBR Green PCR reaction containing 1X SYBR Green PCR master mix (Applied Biosystems, Thermo Fisher Scientific, Inc.), $8 \mathrm{ng}$ cDNA, and $10 \mathrm{nM}$ forward and reverse primers (Qiagen $\mathrm{GmbH}$ ), which were synthesized with the sequences listed in Table I. The reaction conditions were $95^{\circ} \mathrm{C}$ for $10 \mathrm{~min}$ followed by 40 cycles of $95^{\circ} \mathrm{C}$ for $15 \mathrm{sec}$, $60^{\circ} \mathrm{C}$ for $1 \mathrm{~min}$ and $72^{\circ} \mathrm{C}$ for $30 \mathrm{sec}$. Dissociation curves were generated to ensure that a single and specific product was amplified. Quantification cycle values $(\mathrm{Cq})$ were analyzed using SDS2.4 software (Applied Biosystems; Thermo Fisher Scientific, Inc.).

AA hydroxylation assay of hepatic and renal microsomes. The hepatic and renal microsomes were prepared according to the method described previously (12). The conversion of AA was assessed in a reaction mixture of $100 \mathrm{mM}$ potassium phosphate buffer (pH 7.4) containing $3.3 \mathrm{mM} \mathrm{MgCl}_{2}, 80 \mu \mathrm{M}$ AA (Cayman Chemical Company), 1 mM NADPH (Roche Applied Science, Basel, Switzerland) and $0.6 \mu \mathrm{g} / \mu 1$ mouse renal microsomes. Following vortexing and $5 \mathrm{~min}$ preincubation at $37^{\circ} \mathrm{C}, \mathrm{NADPH}$ was added to start the reaction at $37^{\circ} \mathrm{C}$ for $30 \mathrm{~min}$. The reaction was terminated by acidification with formic acid to $\mathrm{pH} 3.5$, following which the production was measured using liquid chromatography-tandem mass spectrometry (LC-MS/MS) following the addition of $2 \mathrm{ng}$ of 20-HETE-d6 (Cayman Chemical Company).

Quantification of eicosanoids using LC-MS/MS. The eicosanoids were measured using the API 3200 Q-trap LC-MS/MS System (Applied Biosystems; Thermo Fisher Scientific, Inc.). The livers and kidneys were homogenized in methanol $(0.1 \%$ formic acid), and added to $2 \mathrm{ng}$ 20-HETEd6 internal standard (Cayman Chemical Company). Lipids were extracted using ethyl acetate, dried under nitrogen, resuspended in methanol, and separated on a reversed-phase Symmetry C18 column (3.5 $\mu \mathrm{m} ; 2.1 \times 150 \mathrm{~mm}$; Waters Corporation, Milford, MA, USA.) at a flow rate of $0.2 \mathrm{ml} / \mathrm{min}$ using solvent $\mathrm{A}$ (water, $0.1 \%$ formic acid) and solvent B (acetonitrile:methanol 6:1, 0.1\% formic acid) as follows: 0-2 min, $25 \% \mathrm{~B}$; 2-10 $\mathrm{min}, 25-75 \% \mathrm{~B}$; $10-18 \mathrm{~min}, 75-95 \% \mathrm{~B} ; 18-30 \mathrm{~min}, 95 \% \mathrm{~B} ; 30-30.5 \mathrm{~min}, 95-25 \%$ B; 30.5-40 min, $25 \%$ B). The effluent was ionized using negative ion electrospray and quantified by multiple reaction monitoring.

Statistical analysis. All data are expressed as the mean \pm standard deviation. Statistical analyses were performed using SPSS 17.0 software (SPSS, Inc., Chicago, IL, USA). Differences were evaluated using Student's $t$-test and $\mathrm{P}<0.05$ was considered to indicate a statistically significant difference. All experiments were performed in triplicate. 
Table I. Primer sequences for reverse transcription-quantitative polymerase chain reaction analysis.

\begin{tabular}{ll}
\hline Gene & \multicolumn{1}{c}{ Sequence } \\
\hline Cyp2c29 & F: 5'-GGCCTCAAAGCCTACTGTCA-3' \\
& R: 5'-AACGCCAAAACCTTTAATC-3' \\
Cyp2c38 & F: 5'-CACTATGGAGACAGAGGTCTA-3' \\
& R: 5'-CCAAATACAGAGTGAAAACG-3' \\
Cyp2j5 & F: 5'-CAACACTGCGATGGGCTTTG-3' \\
& R: 5'-GACTCTCGGTCAGACAAGCTC-3' \\
Human EPHX2 & F: 5'TCAGCAGGATGGTCACTGAG'3 \\
Rouse Ephx2 & R: 5'CATGTGCTGGGACATCTGAG'3 \\
& F: 5'-TGAACACGCCGTTTATGCC-3' \\
Gapdh & R: 5'-TCTCATCACTGGCTCGGAAG-3' \\
& F: 5'-TGCACCACCAACTGCTTAGC-3' \\
& R: 5'-GGCATGGACTGTGGTCATGAG-3' \\
\hline
\end{tabular}

Cyp, cytochrome P450; EPHX2, epoxide hydrolase 2 cytoplasmic; F, forward primer; $\mathrm{R}$, reverse primer.

Table II. Gene expression of EPHX2 in patients with metabolic syndrome.

\begin{tabular}{lcc}
\hline Gene & Description & Fold change \\
\hline EPHX2 & Epoxide hydrolase 2, cytoplasmic & $6.96^{\mathrm{a}}$ \\
\hline
\end{tabular}

${ }^{\mathrm{a}} \mathrm{P}<0.05$, vs. the control group. Data were analyzed using polymerase chain reaction array data analysis software.

Table III. Eicosanoid quantification using liquid chromatography-tandem mass spectrometry.

\begin{tabular}{lcc}
\hline Eicosanoid & WT & TG \\
\hline Hepatic homogenate (ng/mg pro) & & \\
20-HETE & $0.17 \pm 0.05$ & $0.55 \pm 0.10^{\mathrm{a}}$ \\
EETs & $2.98 \pm 0.13$ & $4.25 \pm 0.77^{\mathrm{a}}$ \\
DiHETEs & $2.74 \pm 0.60$ & $7.92 \pm 1.71^{\mathrm{a}}$ \\
Renal homogenate (ng/mg pro) & & \\
20-HETE & $0.37 \pm 0.14$ & $0.70 \pm 0.13^{\mathrm{a}}$ \\
EETs & $7.43 \pm 0.42$ & $6.64 \pm 0.21^{\mathrm{a}}$ \\
DiHETEs & $0.64 \pm 0.13$ & $1.32 \pm 0.24^{\mathrm{a}}$ \\
\end{tabular}

Data are presented as the mean \pm standard deviation. ${ }^{\mathrm{a}} \mathrm{P}<0.05$, vs. WT mice. WT, wild-type; TG, transgenic; 20-HETE, 20-hydroxyeicosatetraenoic acid; EETs, epoxyeicosatrienoic acids; DiHETEs, dihydroxyeicosatrienoic acids.

\section{Results}

Oxidative stress PCR array assay. As previously reported, the ratio of 20-HETE/EETs is disturbed in $C Y P 4 F 2$ transgenic mice, associated with hypertension and hyperglycemia $(8,15)$, however, the mechanism remains to be

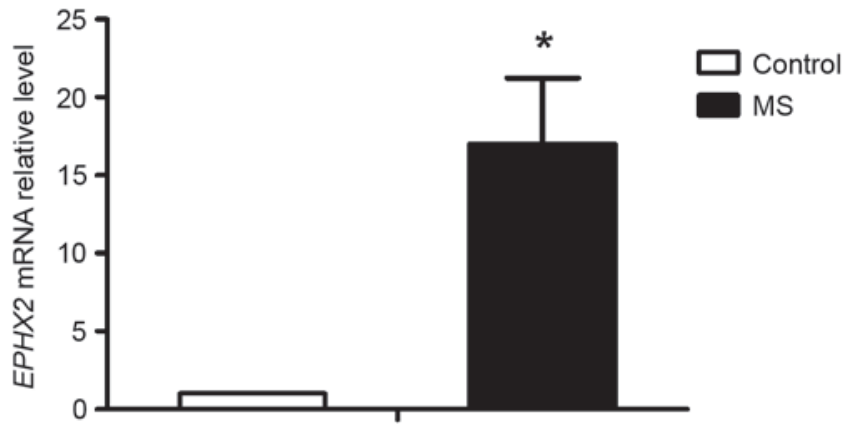

Figure 1. mRNA expression level of $E P H X 2$ in patients with MS. Gene expression levels of $E P H X 2$ were analyzed using reverse transcription-quantitative polymerase chain reaction analysis. GAPDH mRNA was used as an internal control. Data are presented as the mean \pm standard deviation." $\mathrm{P}<0.05$, vs. control group. EPHX2, epoxide hydrolase 2 cytoplasmic; MS, metabolic syndrome.

elucidated. In order to screen out the possible pathogenetic factor, 20 blood samples from an MS group with clinical phenotypes including hypertension, abdominal obesity, diabetes and dyslipidemia, and a control group were collected and analyzed using an oxidative stress PCR array. In the 84 oxidative stress genes, which were evaluated, statistically significant alterations $(>5$-fold; $\mathrm{P}<0.05)$ in the expression of 27 genes were detected in the patients with MS, 24 of which were upregulated and three of which were downregulated (data not shown). Among these genes, the expression of epoxide hydrolase 2, cytoplasmic (EPHX2), coding for the sEH protein was 6.96-fold higher in the MS group, compared with the control group (Table II). This result was also confirmed in the RT-qPCR assay, as shown in Fig. 1, in which the expression of sEH was 17.04-fold higher in the MS group, compared with the control group. sEH, functions to enzymatically hydrolyze EETs (16), which has been implicated in various diseases. In the present study, it was hypothesized that sEH may be important in the disturbed ratio of 20-HETE/EETs in CYP4F2 transgenic mice. 


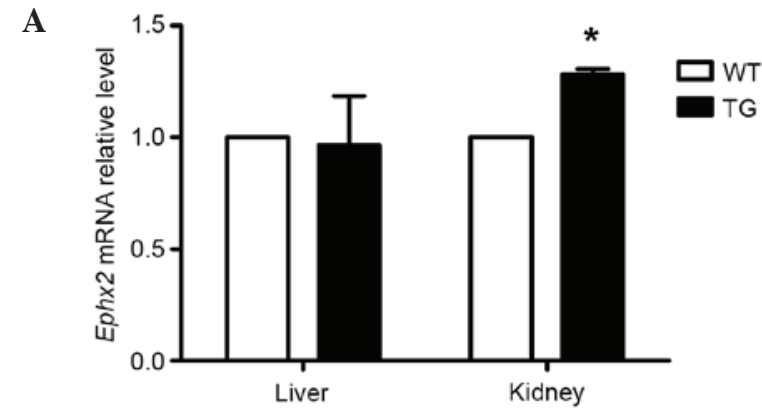

B
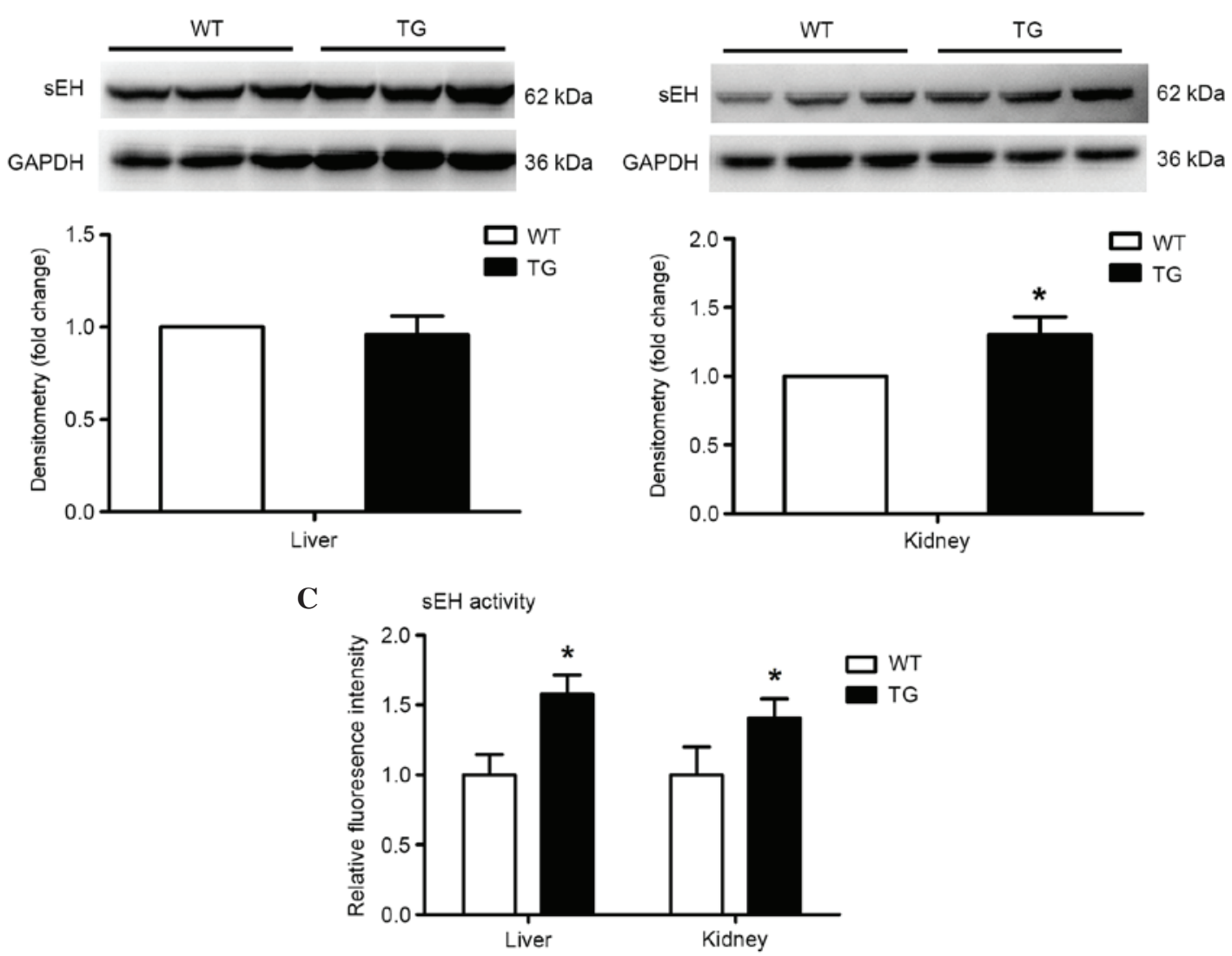

Figure 2. Expression and activity of sEH in $C Y P 4 F 2$ transgenic mice. (A) Reverse transcription-quantitative polymerase chain reaction analysis of mRNA levels of sEH in the liver and kidney. (B) Western blot analysis of sEH in the liver and kidney. (C) sEH activity in the liver and kidney. GAPDH was used as an internal control. Data are presented as the mean \pm standard deviation. ${ }^{*} \mathrm{P}<0.05$, vs. WT mice $(\mathrm{n}=4)$. WT, wild-type; TG, transgenic; CTP4F2, cytochrome P450 4F2; sEH, soluble epoxide hydrolase.

Expression and activity of sEH in CYP4F2 transgenic mice. In order to confirm the association between $\mathrm{SEH}$ and the disturbed 20-HETE/EETs ratio in CYP4F2 transgenic mice, the present study measured the expression of sEH. As shown in Fig. 2A and B, no difference was found in the hepatic expression of sEH between the transgenic and wild-type mice at either the mRNA or protein levels. However, the renal expression of sEH in the transgenic mice was $\sim 30 \%$ higher, compared with that in the wild-type mice. To further examine the alteration of $\mathrm{sEH}$ in the transgenic mice, the activity of sEH was measured using its specific substrate, epoxy fluor 7 . As shown in Fig. 2C, the transgenic mice had higher activity of sEH, compared with the wild-type mice, by $\sim 1.6$-fold and 1.4-fold in the liver and kidney, respectively. Taken together, these results suggested that 20-HETE activated sEH in the liver and kidney.
Eicosanoid quantification using LC-MS/MS analysis. Activated sEH can metabolize EETs into the less bioactive DiHETEs (3). Therefore, the present study measured EETs (14, 15-EET, 11, 12-EET and 8, 9-EET) and DiHETEs (14, 15-DiHETE, 11, 12-DiHETE and 8, 9-DiHETE) in the hepatic and renal homogenates using LC-MSMS (Fig. 3). The CYP4F2 transgenic mice had elevated levels of 20-HETE, compared with the levels in the controls in the liver $(0.55 \pm 0.1$, vs. $0.17 \pm 0.05 \mathrm{ng} / \mathrm{mg}$ pro) and the kidney $(0.70 \pm 0.13$, vs. $0.37 \pm 0.14 \mathrm{ng} / \mathrm{mg}$ pro), as previously reported (15). The total DiHETE levels were markedly higher in the liver (7.92 \pm 1.71 , vs. $2.74 \pm 0.6 \mathrm{ng} / \mathrm{mg}$ pro) and kidney $(1.32 \pm 0.24$, vs. $0.64 \pm 0.13 \mathrm{ng} / \mathrm{mg}$ pro) of the transgenic mice, compared with the wild-type control mice. Of note, the levels of EETs were significantly higher in the liver $(4.25 \pm 0.77$, vs. $2.98 \pm 0.13 \mathrm{ng} / \mathrm{mg}$ pro), but lower in the kidney $(6.64 \pm 0.21$, 

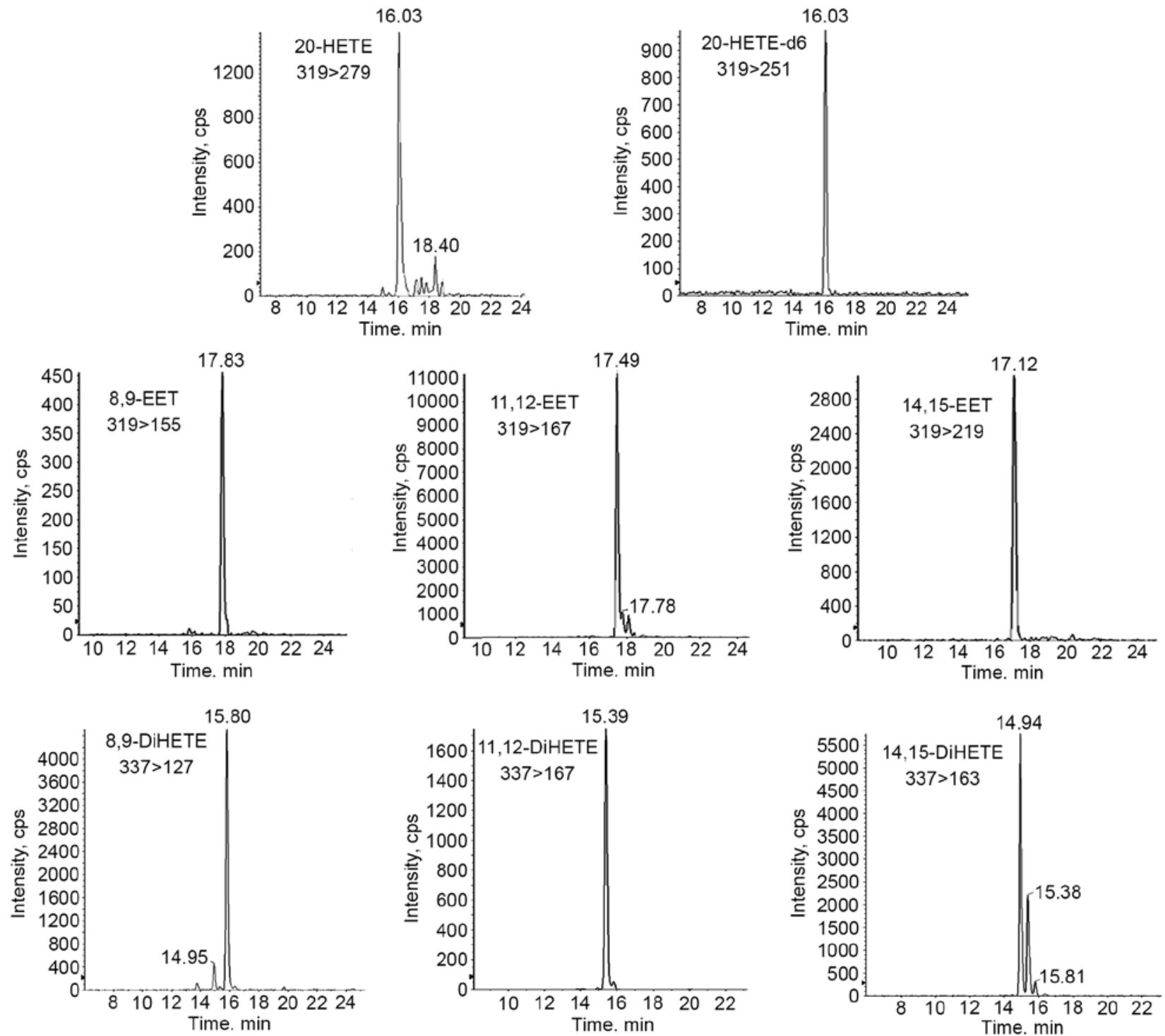

Figure 3. Eicosanoid quantification using liquid chromatography-tandem mass spectrometry. Multiple reaction monitoring was used and the representative chromatograms of eicosanoids are shown, with mass/charge ratio (m/z) indicated. 20-HETE, 20-hydroxyeicosatetraenoic acid; EET, epoxyeicosatrienoic acid; DiHETE, dihydroxyeicosatrienoic acid.

A

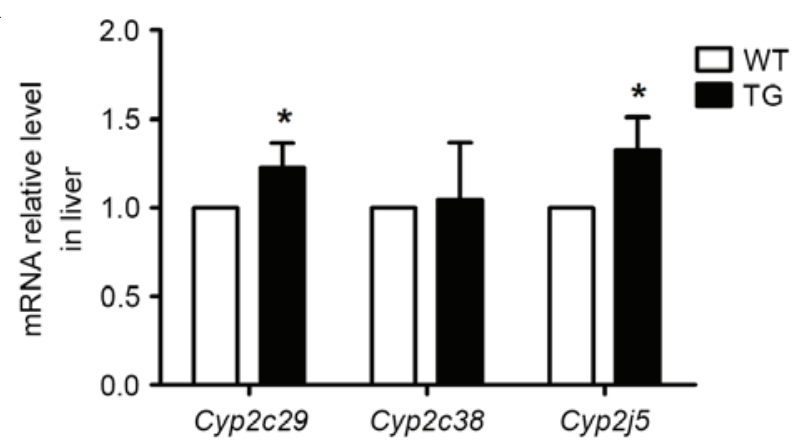

B

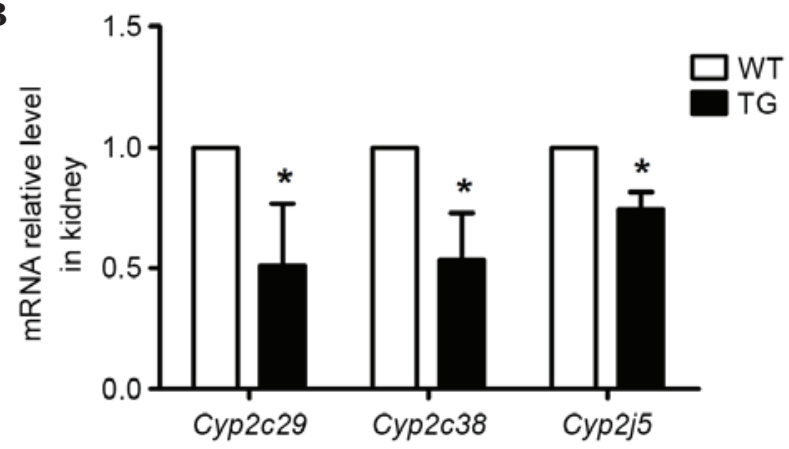

Figure 4. mRNA levels of $C y p 2 c$ and $2 j$ isoforms in $C Y P 4 F 2$ transgenic mice. Reverse transcription-quantitative polymerase chain reaction analysis of mRNA levels of Cyp2c29, Cyp2c38 and Cyp2j5 in the (A) liver and (B) kidney. Gapdh was used as an internal control. Data are presented as the mean \pm standard deviation. ${ }^{*} \mathrm{P}<0.05$, vs. WT mice $(\mathrm{n}=4)$. CYP4F2, cytochrome P450 4FT; WT, wild-type; TG, transgenic.

vs. $7.43 \pm 0.42 \mathrm{ng} / \mathrm{mg}$ pro), compared with the wild-type mice (Table III). These results demonstrated that 20-HETE showed various moderating effects on the generation of
EETs in the liver and kidney, whereas EET degradation was enhanced by 20-HETE-activited sEH in the two tissues. 
A

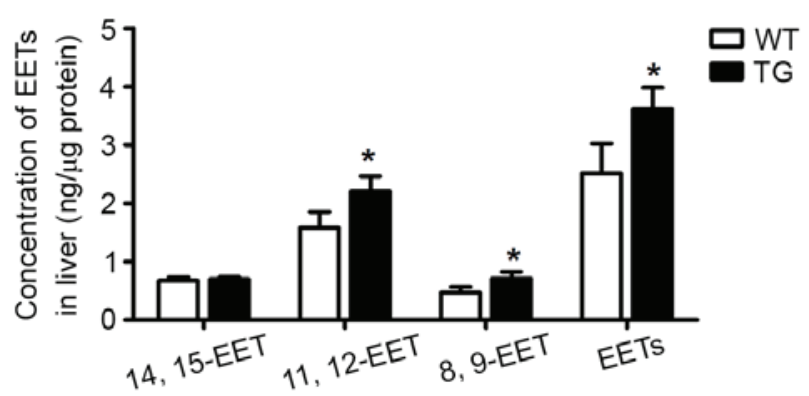

B

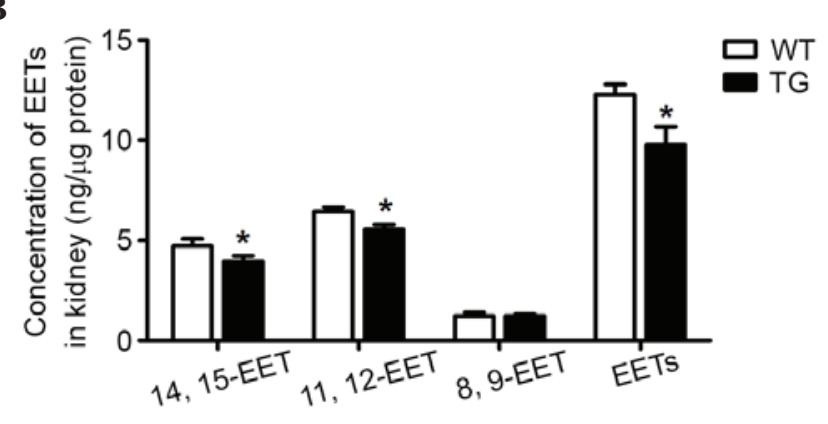

Figure 5. Endogenous EET activity detection using liquid chromatography-tandem mass spectrometry analysis. Endogenous EET activities of the (A) liver and (B) kidney in WT and TG mice. Data are presented as the mean \pm standard deviation. ${ }^{*} \mathrm{P}<0.05$, vs. WT mice (n=6). EET, epoxyeicosatrienoic acid; WT, wild-type; TG, transgenic.

Detection of endogenous epoxygenases, and the expression of Cyp2c29, Cyp2c38 and Cyp2j5 using RT-qPCR analysis. $C y p 2 c 29, C y p 2 c 38$ and $C y p 2 j 5$ are major epoxygenases in the liver and kidney, which can catalyze AA into EETs. To demonstrate whether the endogenous epoxygenase was affected by the high production of 20-HETE in the transgenic mice, the present study measured the mRNA levels of Cyp $2 c 29$, Cyp $2 c 38$ and Cyp2j5 in the liver and kidney using RT-qPCR analysis. As shown in Fig. 4A, the mRNA levels of Cyp2c29 and Cyp2j5 in the transgenic mice were marginally higher in the liver, compared with those in the wild-type mice (by $\sim 20$ and $30 \%$, respectively). However, in the kidney, the mRNA levels of Cyp2c29, Cyp2c38 and Cyp2j5 were decreased in the transgenic mice (by $\sim 50,50$ and $30 \%$, respectively; Fig. 4B). These results demonstrated that the enhanced 20-HETE in the transgenic mice increased the expression of endogenous epoxygenases in the liver and suppressed the expression of endogenous epoxygenases in the kidney.

Detection of endogenous epoxygenase activity using $L C-M S / M S$ analysis. The lack of antibodies for the three isoforms presents a challenge in further detecting the protein expression levels. Therefore, the present study measured endogenous epoxygenase activity in the liver and kidney using LC-MS/MS analysis. Following the incubation of hepatic and nephric microsomes with AA, it was demonstrated that 20-HETE induced the total level of EETs in the liver by 1.2-fold, and caused a 0.8 -fold decrease in the kidney, compared with the wild-type controls (shown in Fig. 5A and B).

\section{Discussion}

As previously reported, the disturbed ratio of renal 20-HETE/EETs is found in CYP4F2 transgenic mice, which is considered to be the molecular basis of hypertension (8). The decrease in EETs may be induced by overdegradation by $\mathrm{sEH}$ or by reduced generation by the endogenous epoxygenases. In the present study, it was demonstrated that 20-HETE activated sEH in the liver and kidney. In addition, 20-HETE showed an opposing action in regulating the endogenous epoxygenases, decreasing endogenous EET synthesis in the kidney and increasing endogenous EET synthesis in the liver.

20-HETE and EETs have been suggested to have vasoactive properties. As they have opposite vascular effects, compared with vasoconstrictors or vasodilators, their proportion determines the vascular resistance and further affects the blood pressure (17). In order to reveal the effect of 20-HETE on AA epoxygenation in the liver and kidney, the present study detected the levels of 20-HETE, EETs and DiHETEs in the hepatic and renal homogenates, respectively. It was found that the overexpression of $C Y P 4 F 2$ in the transgenic mice was associated with higher levels of 20-HETE in the liver and kidney, decreased EETs in the kidney and increased EETs in the liver. EETs, as the major substrate of $\mathrm{sEH}$, can be rapidly metabolized to DiHETEs (11). On the subsequent measurement of the activity of sEH, the present study demonstrated for the first time, to the best of our knowledge, that the 20-HETE increased the protein activity of sEH in the liver and kidney of the hypertensive $C Y P 4 F 2$ transgenic mice. Consistently, previous studies have shown that the protein expression of sEH is upregulated in certain hypertensive models, including two-kidney-one-clip and angiotensin-II infusion hypertension (18-20), however, the mechanism remains to be elucidated. It has been reported that proinflammatory cytokines elevate the protein level of sEH (13). As 20-HETE can induce the expression of cellular adhesion molecules and cytokines (21), the present study hypothesized that 20-HETE-induced inflammation may be the reason for the increased protein level and activity of sEH. By contrast, EETs have important biological effects on anti-hypertension and anti-inflammation. The inhibition of sEH can enhance the beneficial properties of EETs, and has been regarded as a possible treatment for cardiovascular diseases and hyperglycemia $(16,22)$.

The present study demonstrated that the increased DiHETEs in the $C Y P 4 F 2$ transgenic mice was due to the enhanced degradation of EETs via sEH. Although the activation of $\mathrm{sEH}$ explains the higher level of DiHETEs in the transgenic mice, the altered levels of EETs was not caused solely by excessive degradation. It has been reported that Cyp4a14-knockout mice present with a hypertensive phenotype, which is caused by the high levels of Cyp4a12 and 20-HETE in the kidney (23). Our previous study (8) found that the transgenic mice with high expression levels of CYP4F2 showed significantly suppressed levels of all homologous Cyp $4 a$ mRNAs, which suggested an interaction exists between the different isoforms of 20-HETE synthesis. Therefore, the present aimed to investigate whether an interaction exists between 20-HETE and endogenously expressed EETs. The mRNA levels of the three endogenously 
expressed EETs, Cyp2c29, Cyp2c38 and Cyp2j5, which show high levels of expression in the liver and kidney $(24,25)$ were examined. The data demonstrated that the transgene, $C Y P 4 F 2$, had markedly reduced expression levels of Cyp $2 c 29$, Cyp $2 c 38$ and Cyp2j5 in the kidney, but not in the liver, at the mRNA level (Fig. 4A and B). The lack of antibodies of the three isoforms presents a challenge for the further investigations. To investigate the activity of the three endogenous epoxygenases, hepatic and nephric microsomes were separated and incubated with AA in vitro. As shown in Fig. 5, total EET production was markedly lower in the kidney and marginally higher in the liver of transgenic mice, compared with the wild-type controls. The discrepancy in regulatory effects of 20-HETE in different tissues has also been reported in previous studies; 20-HETE acts as a potent constrictor of renal and dilator of bovine coronary arteries (26). In the kidney, 20-HETE has been found to decrease cortical blood flow and increase medullary blood flow (27).

In conclusion, the results of the present study demonstrated that $C Y P 4 F 2$ transgenic mice, with high levels of 20-HETE, increased the degradation of endogenous EETs by activating sEH in the liver and kidney. 20-HETE suppressed endogenous epoxygenases in the kidney, but not in liver. The mechanism underlying the 20-HETE-induced differential regulation of endogenous epoxygenase activity in the liver and kidney requires further investigation. The effects of EETs in cardiovascular physiology are vasodilatory, anti-inflammatory and anti-apoptotic, which are opposite to that of the function of 20-HETE (4-7). Therefore, it was inferred that the amplified disturbed ratio of 20-HETE/EETs in CYP4F2 transgenic mice may further lead to hypertension.

\section{Acknowledgements}

The authors would like to thank Professor Bruce D. Hammock (Department of Entomology and UCD Cancer Research Center, University of California) for providing the sEH inhibiter. This study was supported by the National Natural Science Foundation of China (grant no. 81270343) and the Ministry of Education of China (grant no. 20122104110020).

\section{References}

1. Brash AR: Arachidonic acid as a bioactive molecule. J Clin Invest 107: 1339-1345, 2001.

2. Roman RJ: P-450 metabolites of arachidonic acid in the control of cardiovascular function. Physiol Rev 82: 131-185, 2002.

3. Shen HC: Soluble epoxide hydrolase inhibitors: A patent review. Expert Opin Ther Pat 20: 941-956, 2010.

4. Miyata N and Roman RJ: Role of 20-hydroxyeicosatetraenoic acid (20-HETE) in vascular system. J Smooth Muscle Res 41: 175-193, 2005.

5. Campbell WB: New role for epoxyeicosatrienoic acids as anti-inflammatory mediators. Trends Pharmacol Sci 21: 125-127, 2000.

6. Chen JK, Capdevila J and Harris RC: Cytochrome p450 epoxygenase metabolism of arachidonic acid inhibits apoptosis. Mol Cell Biol 21: 6322-6331, 2001.

7. Zhang Y, Oltman CL, Lu T, Lee HC, Dellsperger KC and VanRollins M: EET homologs potently dilate coronary microvessels and activate $\mathrm{BK}(\mathrm{Ca})$ channels. Am J Physiol Heart Circ Physiol 280: H2430-H2440, 2001.

8. Liu X, Wu J, Liu H, Lai G and Zhao Y: Disturbed ratio of renal 20-HETE/EETs is involved in androgen-induced hypertension in cytochrome P450 4F2 transgenic mice. Gene 505: 352-359, 2012.
9. Certíková Chábová V, Kramer HJ, Vanecková I, Thumová M, Skaroupková P, Tesar V, Falck JR, Imig JD and Cervenka L: The roles of intrarenal 20-hydroxyeicosatetraenoic and epoxyeicosatrienoic acids in the regulation of renal function in hypertensive Ren-2 transgenic rats. Kidney Blood Press Res 30: 335-346, 2007.

10. Eid S, Maalouf R, Jaffa AA, Nassif J, Hamdy A, Rashid A, Ziyadeh FN and Eid AA: 20-HETE and EETs in diabetic nephropathy: A novel mechanistic pathway. PLoS One 8: e70029, 2013.

11. Yu Z, Xu F, Huse LM, Morisseau C, Draper AJ, Newman JW, Parker C, Graham L, Engler MM, Hammock BD, et al: Soluble epoxide hydrolase regulates hydrolysis of vasoactive epoxyeicosatrienoic acids. Circ Res 87: 992-998, 2000.

12. Liu X, Zhao Y, Wang L, Yang X, Zheng Z, Zhang Y, Chen F and Liu H: Overexpression of cytochrome P450 4F2 in mice increases 20-hydroxyeicosatetraenoic acid production and arterial blood pressure. Kidney Int 75: 1288-1296, 2009.

13. National Research Council (US) Committee for the Update of the Guide for the Care and Use of Laboratory Animals: Guide for the Care and Use of Laboratory Animals. 8th edition. National Academies Press, Washington (DC), USA, 2011.

14. Liu Y, Dang H, Li D, Pang W, Hammock BD and Zhu Y: Inhibition of soluble epoxide hydrolase attenuates high-fat-diet-induced hepatic steatosis by reduced systemic inflammatory status in mice. PLoS One 7: e39165, 2012.

15. Lai G, Wu J, Liu X and Zhao Y: 20-HETE induces hyperglycemia through the cAMP/PKA-PhK-GP pathway. Mol Endocrinol 26: 1907-1916, 2012.

16. Imig JD and Hammock BD: Soluble epoxide hydrolase as a therapeutic target for cardiovascular diseases. Nat Rev Drug Discov 8: 794-805, 2009.

17. Singh $H$ and Schwartzman ML: Renal vascular cytochrome P450-derived eicosanoids in androgen-induced hypertension. Pharmacol Rep 60: 29-37, 2008.

18. Imig JD, Zhao X, Capdevila JH, Morisseau C and Hammock BD: Soluble epoxide hydrolase inhibition lowers arterial blood pressure in angiotensin II hypertension. Hypertension 39: 690-694, 2002.

19. Kopkan L, Husková Z, Sporková A, Varcabová Š Honetschlägerová Z, Hwang SH, Tsai HJ, Hammock BD, Imig JD, Kramer HJ, et al: Soluble epoxide hydrolase inhibition exhibits antihypertensive actions independently of nitric oxide in mice with renovascular hypertension. Kidney Blood Press Res 35: 595-607, 2012.

20. Zhao X, Yamamoto T, Newman JW, Kim IH, Watanabe T, Hammock BD, Stewart J, Pollock JS, Pollock DM and Imig JD: Soluble epoxide hydrolase inhibition protects the kidney from hypertension-induced damage. J Am Soc Nephrol 15: 1244-1253, 2004.

21. Ishizuka T, Cheng J, Singh H, Vitto MD, Manthati VL, Falck JR and Laniado-Schwartzman M: 20-Hydroxyeicosatetraenoic acid stimulates nuclear factor-kappaB activation and the production of inflammatory cytokines in human endothelial cells. J Pharmacol Exp Ther 324: 103-110, 2008.

22. Luo P, Chang HH, Zhou Y, Zhang S, Hwang SH, Morisseau C, Wang CY, Inscho EW, Hammock BD and Wang $\mathrm{MH}$ : Inhibition or deletion of soluble epoxide hydrolase prevents hyperglycemia, promotes insulin secretion, and reduces islet apoptosis. J Pharmacol Exp Ther 334: 430-438, 2010.

23. Fidelis P, Wilson L, Thomas K, Villalobos M and Oyekan AO: Renal function and vasomotor activity in mice lacking the Cyp4a14 gene. Exp Biol Med (Maywood) 235: 1365-1374, 2010.

24. Athirakul K, Bradbury JA, Graves JP, DeGraff LM, Ma J, Zhao Y, Couse JF, Quigley R, Harder DR, Zhao X, et al: Increased blood pressure in mice lacking cytochrome P450 2J5. FASEB J 22: 4096-4108, 2008.

25. Luo G, Zeldin DC, Blaisdell JA, Hodgson E and Goldstein JA: Cloning and expression of murine CYP2Cs and their ability to metabolize arachidonic acid. Arch Biochem Biophys 357: 45-57, 1998.

26. Pratt PF, Falck JR, Reddy KM, Kurian JB and Campbell WB: 20-HETE relaxes bovine coronary arteries through the release of prostacyclin. Hypertension 31: 237-241, 1998.

27. Oyekan AO: Differential effects of 20-hydroxyeicosatetraenoic acid on intrarenal blood flow in the rat. J Pharmacol Exp Ther 313: 1289-1295, 2005. 